\title{
Left Hemiparesis in Acute Myeloblastic Leukemia with High Mortality
}

\author{
Edward Kurnia Setiawan Limijadi ${ }^{1 \star}$, Noegroho Harbani ${ }^{2}$, Nani Widorini ${ }^{3}$, Imam Budiwijono ${ }^{1}$ \\ ${ }^{1}$ Department of Clinical Pathology, Medical Faculty, Diponegoro University, Semarang, Indonesia; ${ }^{2}$ Department of Neurology, \\ Ajibarang Government Hospital, Banyumas, Central Java, Indonesia; ${ }^{3}$ Department of Internal Medicine, Ajibarang Government \\ Hospital, Banyumas, Central Java, Indonesia
}

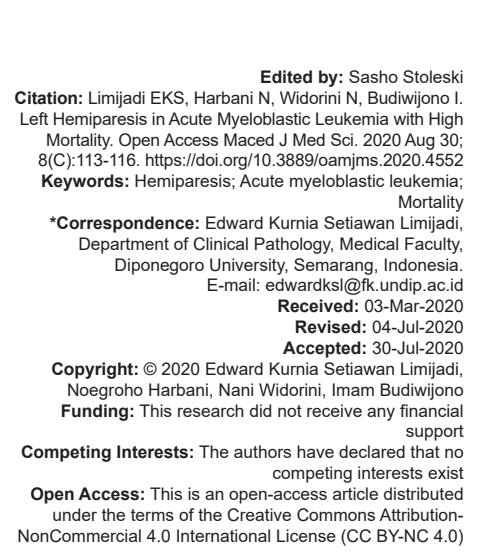

Abstract

BACKGROUND: Leukemia is a blood malignancy that has a variety of types with a variety of clinical manifestations in the body organs in each patient. Acute myeloblastic leukemia (AML) occurs more frequently in adults with clinica manifestations in the central nervous system, especially on the M4 and M5 subtypes.

CASE REPORT: A 42-year-old woman came with a complaint of sudden left hemiparesis. The laboratory results obtained leukocytosis, normochromic normocytic anemia, and thrombocytopenia. Peripheral blood smear found immature cells of the myeloblasts and monoblasts series and monocytosis.

CONCLUSION: The conclusion of the clinical diagnosis and laboratory of the patient is left hemiparesis caused by AML with the suspect of subtype M4 or M5. Patients died within a few hours later, so bone marrow puncture and brain fluid retrieval for malignant cell analysis could not be performed. Brain fluid analysis is important to be performed to enforce the diagnosis of cerebral leukemia.

\section{Introduction}

Leukemia is one of the malignancies in which its incidence began to increase. This is due to the increasing number of risk factors triggering the occurrence of genetic mutation or imbalance between oncogenes and antioncogenes. One of the types of leukemia occurs from the myeloid pathway, better known as acute myeloblastic leukemia (AML) [1].

In 2010, this type of AML reached an incidence of 4.0 (in 100,000 residences) in the US, while in other developed countries such as Germany reached 5.2. This development concluded 35,000 new cancer cases per year. The mortality rate of $A M L$ in the US reached 12,000 in 2015 . The median age of the diagnosed AML was 67 years and there were no differences between males and females [2].

There are seven subtypes of AML based on the French American British (FAB) classification. The M3 subtype in AML has the highest number of incidences, followed by the M2, M0, M4, and M1 subtypes. The other subtypes (M5, M6, and M7) have an incidence rate of less than $10 \%$. The incidence of $A M L$ has various clinical manifestations and a broad spectrum of hematological cell profiles (low to high amounts), including hemoglobin, leukocytes, and platelets [2].

Clinical manifestations of AML may involve the central nervous system called cerebral leukemia which is fatal in patients. Manifestations of the central nervous system have a low prevalence at the time of diagnosing AML. A diagnostic strategy of complications and appropriate treatment is required for the situation. Here will be shown a case report of an AML patient with left hemiparesis as a complication in the central nervous system with high mortality [3], [4].

\section{Patient and Observation}

A 42-year-old woman came to the emergency unit with the main complaint of a sudden inability to move the left hand and leg. The patient went straight to the hospital that morning (at 9 a.m.). The patient also felt difficult to speak, slight fever, and dizziness along with the main complaint. She did not feel either nauseous or vomiting. Defecation and urination were within normal limits. The patient had a history of stroke 
and underwent a routine follow-up to the neurologist. She had no previous history of diabetes mellitus and hypertension, but the patient claimed to have a history of high-fat laboratory results.

Patients seemed conscious in general and appeared weak with the blood pressure of $150 / 90 \mathrm{mmHg}$, pulsation rate of 90 times/min, respiratory rate of 20 times $/ \mathrm{min}$, and temperature of $37.6^{\circ} \mathrm{C}$. The eye examination obtained by mild anemic conjunctiva and there was no icteric sclera. The neurology examination of the face found no abnormalities on the right side either on the forehead, palpebral, tongue, and lips. There were no abnormalities and bleeding from the nose, mouth, nor the ears. Cardiac and lung examinations were within normal limits. The abdominal examination did not find organomegaly or tenderness. There was no lymphadenopathy in the lymph glands. There was decreased muscle strength in the left upper and lower extremities compared to the right extremities. The physiological reflex examination on the left side of the body increased as well as pathological reflexes, while the right side of the body found normal physiological reflexes and negative pathological reflexes.

Laboratory examination obtained a hemoglobin level of $10 \mathrm{~g} / \mathrm{dL}$ comparable to the decreased hematocrit level, a corresponding number of erythrocytes to the hemoglobin, normal $\mathrm{MCV}$, and $\mathrm{MCH}$ levels, indicating normochromic normocytic anemia. It also found thrombocytopenia with a platelet count of $130,000 /$ $\mu \mathrm{l}$. There was an increased number of leukocytes of $78.000 / \mu \mathrm{l}$. The patient's laboratory examination results were summarized in Table 1.

Table 1: The complete result of laboratory examination of the patient

\begin{tabular}{ll}
\hline Parameter (values) & Laboratory results \\
\hline Hematological parameters & \\
Hb $(\mathrm{g} / \mathrm{dL})$ & 10.0 \\
Hct $(\%)$ & 32.0 \\
Erythrocyte number $\left(\times 10^{6} / \mu \mathrm{l}\right)$ & 3.4 \\
MCV $(\mathrm{FI})$ & 93.2 \\
$\mathrm{MCH}(\mathrm{Pg})$ & 29.3 \\
MCHC $(\%)$ & 30.5 \\
Leukocyte number $\left(\times 10^{3} / \mu \mathrm{l}\right)$ & 78 \\
Platelet number $\left(\times 10^{3} / \mu \mathrm{l}\right)$ & 130 \\
Clinical chemistry & \\
Random blood glucose $(\mathrm{mg} / \mathrm{dL})$ & 350 \\
AST $(\mathrm{IU} / \mathrm{L})$ & 43 \\
ALT $(\mathrm{IU} / \mathrm{L})$ & 35 \\
Ureum $(\mathrm{mg} / \mathrm{dL})$ & 25 \\
Creatinine $(\mathrm{mg} / \mathrm{dL})$ & 1.1 \\
\hline
\end{tabular}

The peripheral blood morphology obtained normochromic normocytic anemia. The estimated number of leukocytes increased with the appearance of blast cells of $13 \%$, AMC of $20 \%$, neutropenia, and monocytosis as well as giant lysosomes (Figure 1). It also found a decreased estimated number of platelets with large platelet size.

The patient has been declared stable with monitoring in the emergency room while waiting for the head CT-scan in the referral hospital. The patient experienced a sudden loss of consciousness at 2.45 p.m. and underwent cardiac pulmonary resuscitation. The patient was declared dead at 3 p.m. in the emergency

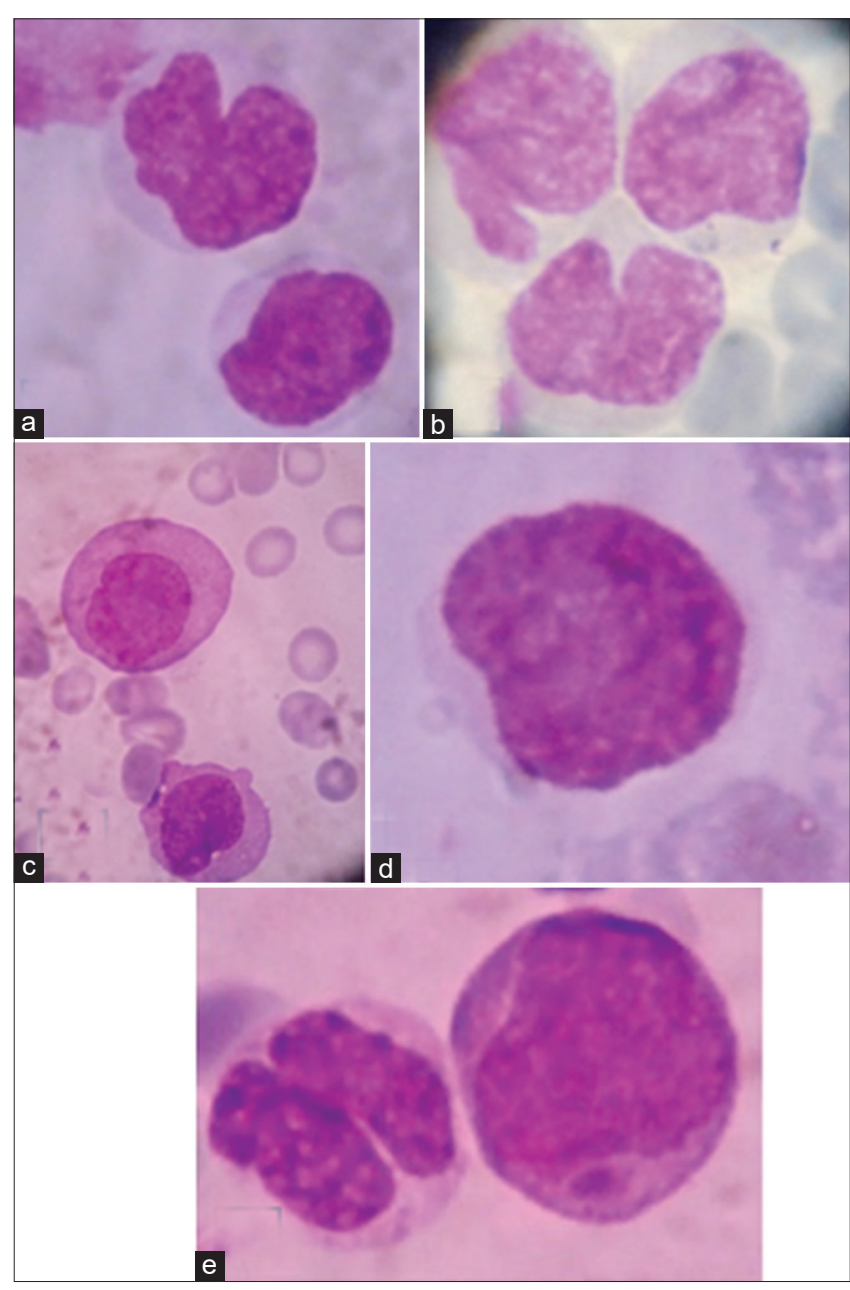

Figure 1: Cells found in peripheral blood smear. Explanation: (a-c) Monoblast; (d) myeloblasts; (e) myeloblast with giant lysosome (arrow)

room. There was not enough time for performing bone marrow puncture to establish the definite diagnosis of the hematological malignancy.

\section{Discussion}

The patient came with symptoms and clinical signs of lateralization, leading to a diagnosis of stroke. Complete blood count examination showed bicytopenia (normochromic normocytic anemia and thrombocytopenia) and leukocytosis. The peripheral blood smear showed normochromic normocytic anemia, thrombocytopenia, and blasts cells (myeloblasts and monoblasts) accompanied by neutropenia and monocytosis. Clinical chemistry examination showed normal liver and kidney function and hyperglycemia.

The diagnosis was established based on clinical findings of left hemiparesis and laboratory results, suspecting cerebral leukemia caused by suspected AML. The patient also experienced reactive hyperglycemia with a differential diagnosis of Type 
2 diabetes mellitus. The patient underwent a routine follow-up to the neurologist due to her previous history of stroke. However, the history of her complete blood count was within normal limit and indicated no such condition. The state of hyperglycemia in the patient was suspected reaction of an acute inflammatory condition and the patient claimed to have no prior diabetes mellitus history; however, the differential diagnosis of Type 2 diabetes mellitus was taken for being evaluated subsequently.

The clinical course of acute leukemia is worse than chronic leukemia. Various symptoms can arise in acute leukemia patients that vary from each person. Some patients came with mild to severe symptoms; some others came with sudden or long-lasting symptoms. The symptoms and the clinical signs include lethargy, fever, decreased appetite, decreased weight, enlarged stomach, bleeding, visual disturbances, headaches, and others [5], [6].

Laboratory examination obtained in acute leukemia patients showed various results. In general, it showed leukocytosis accompanied by anemia with platelets. Some cases did not indicate such a condition but showing normal leukocytes, anemia, and thrombocytopenia. There were cases of leukemia with a normal state of blood cells called aleukemic leukemia. The results of the laboratory examination obtained should be adjusted to the patient's clinical condition. The subsequent laboratory test carried out is the peripheral blood smear to perform screening and bone marrow puncture to establish the diagnosis of acute leukemia [5], [7], [8].

The peripheral blood smear was used for the evaluation of the patient's cells, both for screening and diagnosing hematological disorders. Various cells can be found depending on the type of leukemia. Myeloblasts and lymphoblasts, as well as other immature cells from various maturation pathways of blood cells, can be found depending on the type and subtype of leukemia [7], [8]. Definite diagnosis was established by performing bone marrow puncture as well as bone marrow biopsy. The parameters of the results were the cellularity, the dominant cell types in the hematopoietic pathway underlying leukemia, and establishing the diagnosis of leukemia.

One of the acute leukemia types found is AML developing from the myeloid progenitor cells. According to the FAB classification, this type of leukemia is divided into eight subtypes from M0 to M7. Each subtype of AML has its distinguished characteristic based on the findings of immature cells and the development of the cells. This is the underlying diagnosis of the AML subtype.

In this case, the peripheral blood smear showed myeloblasts and monoblasts despite not performing the bone marrow puncture. This is the underlying diagnosis of the suspected AML of subtypes M4 and
M5. Furthermore, the patient was showing a symptom of hemiparesis, which is a manifestation of leukemia cells in the central nervous system. This condition is supported by Stefano et al. (2005) [9] stated that the complications of AML to the central nervous system could occur in AML, mostly in M3 subtype followed by other subtypes such as M4, M5, and M1. Other authors suggested the same opinion. Manifestations of the central nervous system could be obstruction, bleeding, infection, and infarction. This condition could occur due to the hyperviscosity of blood, leading to the occlusion of the brain veins. Examination of cerebrospinal fluid analysis became important to establish the diagnosis of the complications called cerebral leukemia by discovering the presence of foreign or malignant cells [10].

\section{Conclusion}

The condition of acute leukemia, especially AML with a subtype of M4 and M5 known from the peripheral blood smear supported by the results of bone marrow puncture, required immediate LCS examination to establish the diagnosis of cerebral leukemia. This is done for the treatment of leukemia patients and lowering mortality in leukemia patients due to the abnormalities in the central nervous system.

\section{References}

1. Kavianpour M, Ahmadzadeh A, Shahrabi S, Saki N. Significance of oncogenes and tumor suppressor genes in AML prognosis. Tumour Biol. 2016;37(8):10041-52. https://doi.org/10.1007/ s13277-016-5067-1

PMid:27179964

2. Sitelbanat HO, Kordofani AA, Osman IM, Musa OH, Altayb HN Elamin BK. Prevalence of the diffrent FAB sub type of acute myeloid leukemia related to hematological parameters in Sudanese. J Hematol Blood Disord. 2017;3(1):1-5. https://doi. org/10.15744/2455-7641.3.102

3. Alakel N, Stölzel F, Mohr B, Kramer M, Oelschlägel U, Röllig C et al. Symptomatic central nervous system involvement in adult patients with acute myeloid leukemia. Cancer Manag Res. 2017;9:97. https://doi.org/10.2147/cmar.s125259

PMid:28435324

4. Shihadeh F, Reed V, Faderl S, Medeiros LJ, Mazloom A Hadziahmetovic $M$, et al. Cytogenetic profile of patients with acute myeloid leukemia and central nervous system disease. Cancer. 2012;118(1):112. https://doi.org/10.1002/cncr.26253

5. Shahab F, Raziq F. Clinical presentations of acute leukemia. J Coll Physicians Surg Pak. 2017;24(7):472-6.

PMid:25052968

6. Goy J, Smith T, Nantel S, Mourad YA, Broady R, Narayanan S, et al. The clinical and diagnostic pathway for adults with acute leukemia in BC. BC Med J. 2017;59(1):22-8. 
7. Davis AS, Vierra AJ, Mead MD. Leukemia: An overview for primary care. Am Fam Physician. 2014;89(9):731-8.

8. Faivdullah L, Azahar F, Htike ZZ, Wei YN. Leukemia detection from blood smears. J Med Bio Eng. 2015;4(6):488-91. https:// doi.org/10.12720/jomb.4.6.488-491

9. Stefano VD, Sora F, Rossi E, Chiusolo P, Laurenti L, Fianchi L, et al. The risk of thrombosis in patients with acute leukemia: Occurence of thrombosis at diagnosis and during treatment. J Thromb Haemost. 2005;3(9):1985-92. https://doi. org/10.1111/j.1538-7836.2005.01467.x

PMid:16102104

10. Algharras AA, Mamourian A, Coyne T, Mohan S. Leukostasis in an adult with $\mathrm{AML}$ presenting as multiple high attenuation brain masses on CT. J Clin Diagn Res. 2013;7(12):3020-2. https://doi. org/10.7860/jcdr/2013/6638.3893

PMid:24551717 